\title{
A history of crowd simulation: the past, evolution, and new perspectives
}

\author{
Soraia Raupp Musse ${ }^{1}\left[\right.$ D $\cdot$ Vinicius Jurinic Cassol $^{2} \cdot$ Daniel Thalmann $^{3}$
}

Accepted: 1 July 2021 / Published online: 5 August 2021

(c) The Author(s), under exclusive licence to Springer-Verlag GmbH Germany, part of Springer Nature 2021

\begin{abstract}
This paper aims to discuss the past, evolution, and new perspectives in crowd simulation. Many work have been produced and published in this area that was launched approximately 30 years ago. In this paper, we re-visited the main aspects of the area, presenting the periods and evolution we had in the past. In addition, we also discuss the present and possible trends for the future.
\end{abstract}

Keywords Crowd simulation · Virtual humans · Behavioral animation

\section{Introduction}

To have a credible application using crowds in virtual environments, several aspects of the simulation must be addressed. The most relevant are environment modeling, behavioral animation, and crowd rendering [70]. Such aspects are essential in order to provide a positive impact of performed simulations. However, some of them may be more or less relevant depending on the application. If there is no appropriate environment model, the characters will not behave accordingly, as they can perform actions in the wrong places or not performing at all. Serious simulations like evacuation scenarios are examples of the relevance of credible and realistic environments. If there is no satisfactory rendering, even the best behavior model will not be compelling. In this case, we can mention games and movies, where the visual impact is undoubtedly essential. However, computer animation to be used in training applications should also take care of visual aspects to provide better immersion and user experience. If there is no good behavioral model, even a simulation

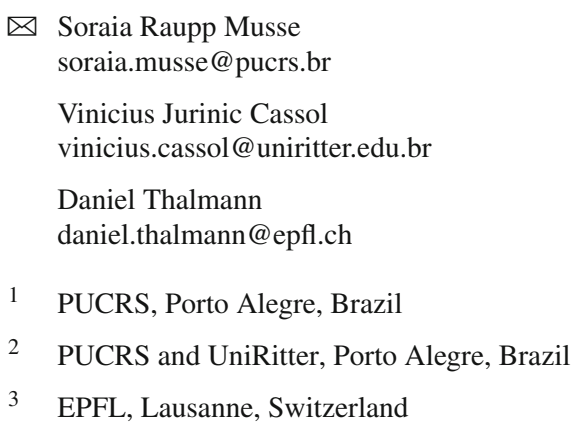

using the best rendering method will not look believable just after few seconds. Finally, interactive applications must simulate and display a varied ensemble of virtual characters and their behaviors efficiently. Rendered characters must visually adjust to the environment in terms of visual reactions, i.e., they must be affected by light and other effects in the same way as their surroundings. Therefore, when considering crowd simulation, the effort to be made is actually a compromise between many aspects (rendering, animation, behavior, frame rates) and the purpose of the application.

Since the seminal paper by Craig Reynolds [88], where he proposes a way to control and animate groups of entities as one only entity, many have been achieved in the crowd domain. In this paper, we want to trace the area since 1987, discussing the evolution and problems that have been solved and the critical challenges that are still open. In particular, we focus on crowd simulation techniques, i.e., behaviors, panic situations, flocks, collision avoidance, navigation, path planning, environments when populated with crowds, emotions, psychological aspects, data-driven approaches, personalities and emotions in crowds, and recently methods that use machine learning techniques.

Some crowd surveys have already been proposed. Some of them are mainly focused on crowd analysis, like crowd behaviors studies, as crowd density estimation or counting [40,90,110]. Azahar et al. [3], in 2008, presented the pioneers' authors in crowd simulation, organized in an excellent timeline until 2006, and focus mainly on real-time applications and their challenges. Nasir et al. [73] discuss the crowd domain in a more general way considering environment mod- 
eling, simulation methods, and animation in a shorter paper containing approximately 30 bibliographical references. In 2015, Ijaz et al. [42] presented a survey paper specifically on crowd simulation using hybrid techniques. So, the contribution of the present paper is to analyze more than 300 papers, even if approximately 100 are cited here, regarding the historic context and evolution of papers and methods in crowd simulation, not focused on one specific technique. Although it may be possible that a paper focused on virtual environments or virtual reality is cited in this manuscript, it happens because they contribute with the proposed method to simulate crowds as well, in the authors' point of view. So, this text does not intend to provide a survey on crowd interaction, rendering, virtual environment, parallel processing, even if all these subjects are co-related with the theme. The main reason is that we want to avoid a general text by citing as many methods as possible from the studied papers. Therefore, our goal is to propose a way to see the history and imagine the future with respect specifically to crowd simulation techniques.

We organized all the studied methods in this paper into six main periods: before 2000, from 2000 to 2004, from 2005 to 2009 , from 2010 to 2014 , from 2015 to 2019 , and then papers from 2020 and 2021. Our objective is to highlight the proposals for new methods in these defined periods. It is essential to point out that, in general, we indicated the periods of the proposal of methods, but this does not mean that these methods have not being studied in future periods of time.

\section{Literature review: an analysis of crowd simulation techniques over the past decades}

Firstly, in this section, we describe the methodology applied in this work, i.e., the scope of our systematic review and the selection criteria used to select the papers included in our study. Then we present details about the proposed word clouds used to illustrate each period. After we have presented our methodology, subsequent sections detail each period of research.

\subsection{The Research Methodology}

To proceed with the systematic review, we used search engines at ACM, IEEE, Elsevier, Springer, and Google Scholar. The keywords used are: Crowds and Simulation, and this process results in thousands of papers from which we selected 319 based on the main topic, distributed along the time as shown in Fig. 1.

Due to the high number of papers in crowd simulation and co-related areas as rendering, parallel processing, crowds on GPU, etc., we focused only on papers that describe methods in simulating crowds concerning motion, navigation, colli-

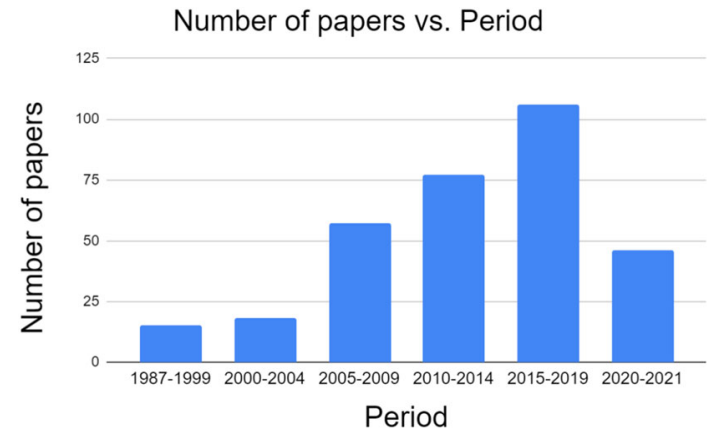

Fig. 1 Distribution of selected 319 papers along the time.

sion avoidance, perception, personality, emotion, and other simulation methods. From such papers, we extracted authors, full references, abstracts, keywords, and titles. Papers were grouped by period, firstly before 2000, and then one period at every 5 years.

We selected some papers to be cited in this work for each period, since we avoid mentioning all 319 selected. The selection of papers was made based on two simple criteria: (1) We tried to include the most known and cited methods in the area presenting diversity in the proposed methodologies, while (2) trying to distribute citations among most relevant and cited authors in the area. From this selection, we have 115 papers cited in this paper.

We use word clouds to illustrate the most mentioned words in the titles of the selected papers. Firstly, it is essential to mention that our goal with word clouds was not to guide the papers we will discuss in this work but only to illustrate the primary terms (probably related to the techniques) proposed at each period. At each period, we focus on the proposals showing how the different ideas emerged as a function. Of course, many techniques has been proposed until nowadays, as different ways to provide collision avoidance, for example. Indeed, we tested word clouds with words from abstracts; however, the words from the title seem to us more interesting because usually the titles are innovative, so new terms and ideas are typically used. Please, note that stop words have been removed ${ }^{1}$. Figure 2 presents a word cloud formed by the conferences and journals where we found such selected papers. The conferences where we found most papers in crowds domain are $\mathrm{MIG}^{2}, \mathrm{SCA}^{3}, \mathrm{IVA}^{4}$ and $\mathrm{CASA}^{5}$, while

\footnotetext{
1 We used framework available in wordart.com to generate the word clouds

2 ACM SIGGRAPH Conference on Motion in Games

3 Symposium on Computer Animation

4 Intelligent Virtual Agents

5 Computer Animation and Social Agents
} 
journals are IEEE CG\&A ${ }^{6}$, IEEE TVCG ${ }^{7}$, Elsevier $C \& G^{8}$, and $\mathrm{ACM} \mathrm{TOG}^{9}$. In the next sections, we describe some of these papers organized in periods, as mentioned before.

\subsection{Crowd papers before 2000: the age of flocks and behaviors}

Reynolds in 1987 [88] proposed the first method to control and animate groups of characters. In his method, he innovated proposing a distributed model where each member of the crowd (called boids) could perceive and react individually to the environment and the other boids. Reynolds suggested the term flock to indicate the immediate group an individual belongs to. Also, boids into a flock interact to avoid collisions as well as to compute speeds and orientations. Tu and Terzopoulos [101] have worked on behavioral animation for creating artificial life, where virtual agents are endowed with synthetic vision and perception of the environment. The repertory of fishes' behaviors relies on their perception of the dynamic environment, and the fishes' reactions are not entirely predictable because they are not scripted.

Helbing et al. [35] proposed a model based on physics and sociopsychological forces to describe human crowd behavior in panic situations. The model is set up by a particle system where each particle has a predefined speed in a specific direction to which it tends to adapt its instantaneous velocity within a certain time interval. Simultaneously, the particles try to keep a velocity-dependent distance from other entities and walls controlled by interaction forces. Musse and Thalmann in 1997 [69] proposed the first method to simulate crowds based on a hierarchical control, having the crowd formed by groups which are formed by individuals. In this case, individuals are homogeneous and interact only with closer agents to avoid collisions. Agents have distributed and known goals generating a goals-based method. Also, in this paper, sociological aspects such as leadership were mathematically formulated to be taken into account in crowd decisions.

In 1999, Reynolds [87] proposes steering behaviors as a solution to model autonomous characters in animation and games by endowing them with the ability to navigate around their world in a life-like and improvisational manner. This paper divides motion behavior into three levels: the middle level of steering behaviors, the lower level of locomotion, and the higher level of goal setting.

Figure 3 shows the word cloud formed by the titles of 15 papers (from selected 319 papers) selected from 1987 to 1999 . We only discarded the term "crowd simulation"

\footnotetext{
${ }^{6}$ IEEE Computer Graphics and Applications

${ }^{7}$ IEEE Transactions on Visualization and Computer Graphics

${ }^{8}$ Elsevier Computers and Graphics

9 ACM Transactions on Graphics
}

because it appears in all periods. As we can see, words as pedestrian, behaviors, flocks, artificial, fishes, schools, herds, and physics illustrate the techniques proposed in this period, where authors firstly think and elaborate about groups of characters. In addition, we notice the presence of words evacuation, human collision locomotion, however, less mentioned in the titles (smaller in the figure), indicating that researchers were starting to work on these issues.

\subsection{Crowd papers from 2000 to 2004: virtual humans behaviors and environments}

Figure 4 presents the word cloud generated as a function of the titles of 18 papers selected in this period. Firstly, it is interesting to see that, after discarding the term "crowd simulation," the most cited term is "virtual human." It contrasts with the last period, where we found in most cases the word pedestrian. It can indicate that researchers, in the last period, used most of the term pedestrians (e.g., [35]), which may be because in computer vision, people refer to pedestrians in video sequences. In the case of calling virtual humans, it may represent that the researchers in this generation focused their attention to animate and simulate individual behaviors. This period is characterized by the definition of crowd structures, behaviors, and the connection with the environments.

Farenc et al. [29] presented an architecture to simulate virtual humans in complex environments. The work proposes to create an environment not only represented with geometry but also with behavioral rules that virtual humans could apply. Lamarche and Donikian [53] propose a navigation algorithm to evolve in a topological structuring of the geometric environment to allow fast pathfinding and an efficient reactive navigation algorithm for virtual humans evolving inside a crowd. Also, in this period, we observe the terms panic, evacuation, escape, steering, and controlling in the word cloud (Fig. 4), showing that researchers were concerned with evacuation techniques. For instance, Helbing et al. [34] in 2000 investigate the mechanisms of panic and jamming caused by the motion in crowds. Their simulations suggest practical ways to prevent dangerous crowd pressures while proposing an optimal strategy for an escape from a smokefilled room. Braun et al. [14] extend the Helbing's model to provide crowd evacuation where agents can rescue others and have different individual intentions and states.

One important word in the cloud, in this period, is "behavior," as we could see many papers proposing ways to model the behavior of crowds, groups, and individuals in this period. One example is the proposed ViCrowds described by Musse and Thalmann [71], where authors propose that a crowd is formed by groups, which in turn are formed by individuals. In this case, users can control each level of the hierarchy, as desired, with different types of information. Constrained animation of flocks proposed by Anderson [2] focuses on 


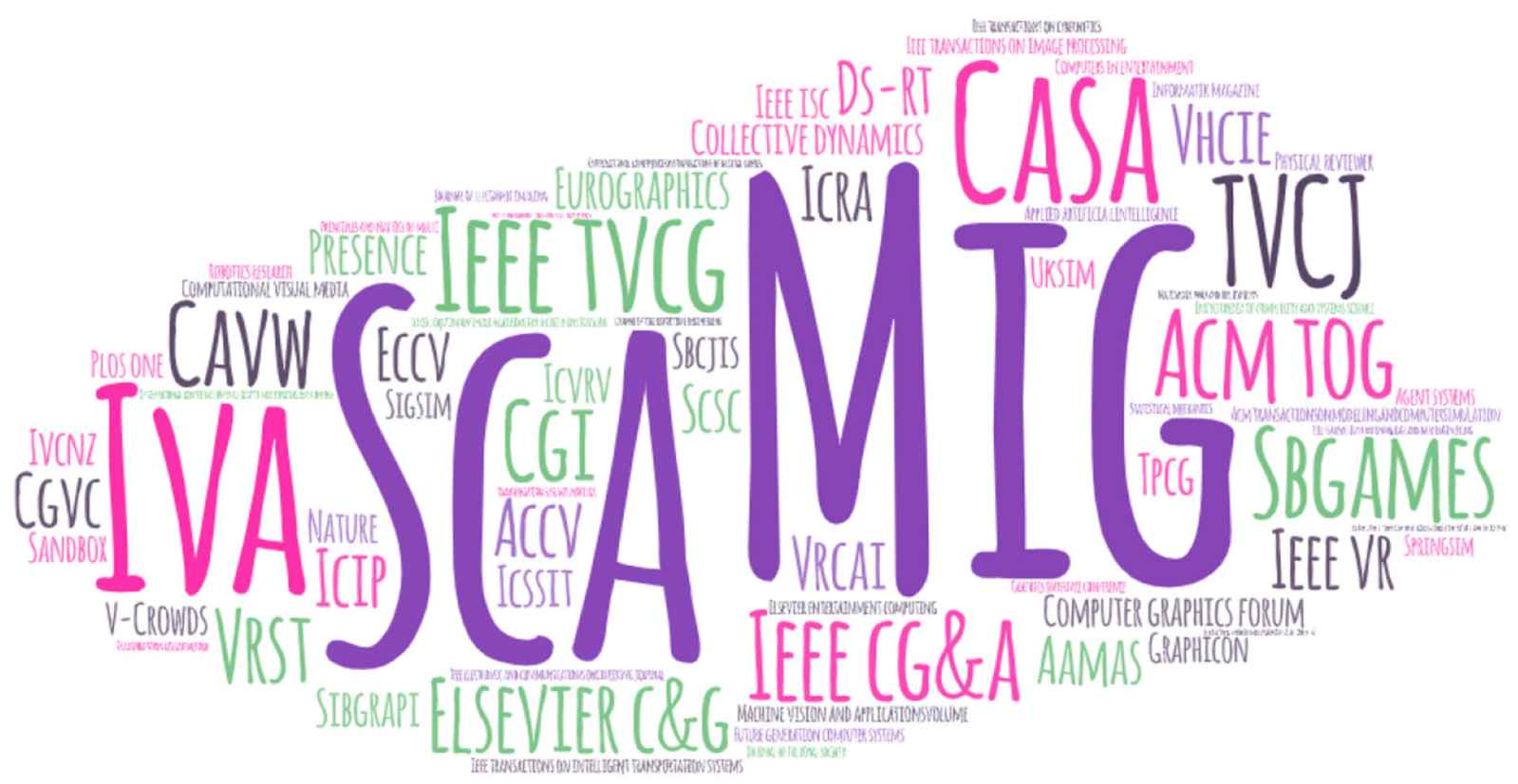

Fig. 2 Journals and conferences from where we selected papers

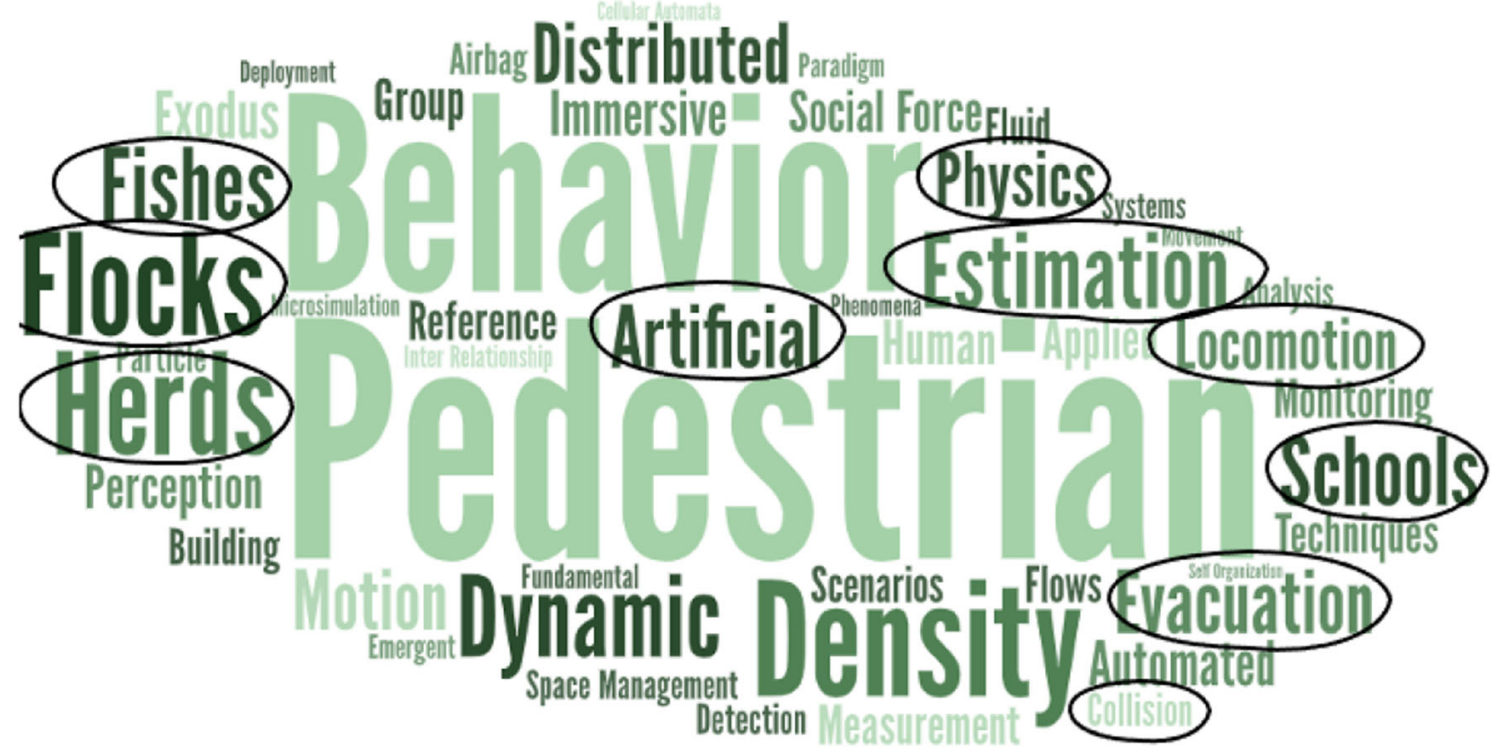

Fig. 3 Main terms collected from 15 papers published between 1987 and 1999. The word cloud is composed by 48 words

the generation of constrained group animations, where users can position constraints on agents' positions at any time in the animation or constrain the entire group to meet the center of mass or shape constraints. Loscos et al. [62] provide, in 2003, a technique that allowed the simulation of up to 10,000 pedestrians walking in real time. The main focus of the work is to present a pedestrian crowd simulation method to improve the local and global reactions of pedestrians.

In this period, the virtual human was the most used term in the 18 papers analyzed after crowd simulation. However, other terms also appeared as Environment, Real, Urban,
Escape, steering, etc. It may indicate that many methods describing the integration between environments and crowds, and how to structure crowds (in terms of groups) have been proposed.

\subsection{Crowd papers from 2005 to 2009: the age of virtual agent navigation and the beginning of data-driven techniques}

In this period, many navigation techniques have been proposed. In 2005, Lai et al. [52] propose the group motion 


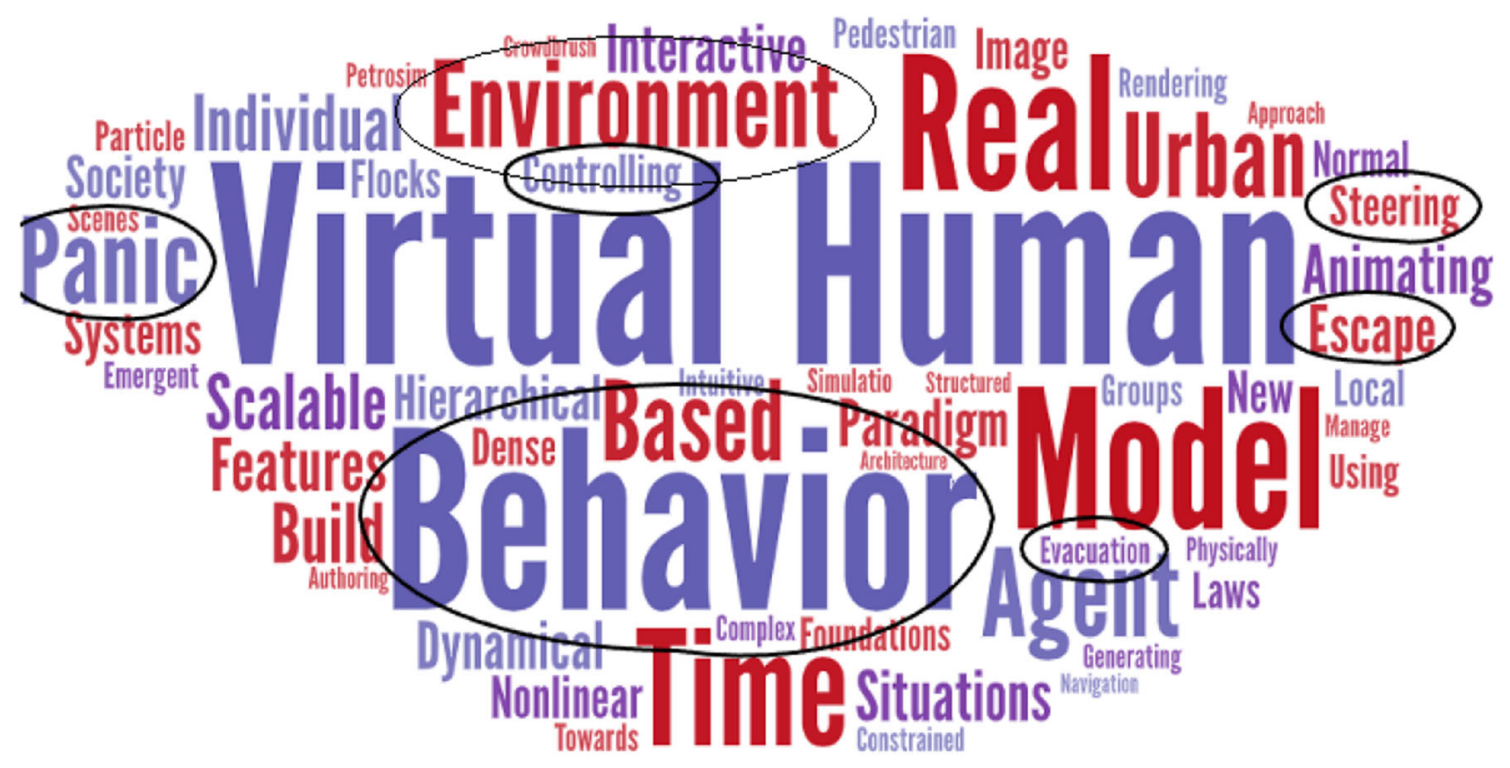

Fig. 4 Main terms collected from 18 papers published between 2000 and 2004. The cloud is composed by 57 different words

graphs, a data-driven animation technique for groups of discrete agents, such as flocks, herds, or small crowds. Sung et al. [96] describe a fast path planner based on probabilistic roadmaps to navigate characters through complex environments. Also, Pettre et al [83] propose a navigation graph to animate crowds in terrains. And, our last example here, from many others (e.g., corridor map [32] and hierarchical path planning [104]), is Continuum Crowds [99], that present a dynamic potential field that integrates global navigation with moving obstacles efficiently, providing the motion of large crowds without the need for explicit collision avoidance. Yersin et al. [109] present an improved short-term collision avoidance algorithm and a simple efficient group behavior for crowds. Rodrigues et al. [89] propose a model for generating groups of characters' steering behaviors based on a biologically motivated space colonization algorithm. In this case, agents into the crowd could apply formations like letters in a word.

Interestingly, 2007 was a year that some researchers propose data-driven methods to simulate crowds. Musse et al. [68] call their method a way to simulate crowds based on computer vision. Indeed, pedestrian trajectories were tracked and used to clustering groups of paths distributed in vector fields to fed virtual humans with motion stimuli. Courty and Corpetti [19] also propose to represent crowd motion as a time series of velocity fields estimated from a real crowd, and Lerner et al. [56] propose that, during a simulation, autonomous agents search for examples taken from real people that closely match the situation that they are facing and guide them in the applied behavior. Also, the last work in data driven, also published in 2007, proposes that agents learn how to move based on observed trajectories [55].

Also, in this period, Pelechano et al. [82] propose incorporating psychological models, roles, and communication in crowd simulation and also model crowds with leaders [81] that could influence the other agents. Cho et al. [18] propose integrating the known BDI (Belief-Desire-Intention) model to simulate crowds. The intention was to allow the characters to perform realistic behavior by adapting their actions with the sensed information in a changing environment. Grillon et al. [33] introduced gaze attention behaviors to crowd animation, while Maim et al. [63] proposed the use of accessories like bags or glasses or even carrying gifts to improve the realism of crowd behaviors.

Other topics have also been proposed in this period as methods for collision avoidance [45,77], to simulate dense crowds [72], and the two first books in crowd simulation have been published in this period by Musse and Thalmann [98] and Pelechano, Allbeck and Badler [79].

This period was very rich in terms of new ideas and insights. Concerning the word cloud illustrated in Fig. 5, we can observe a great diversity of words used in the paper titles. Interestingly, in this period, researchers started to use the term "virtual agent" more than virtual human or pedestrian. It may indicate the search for more autonomous and "intelligent" agents, as it reveals the published papers in the period. Other words highlighted in Fig. 5 are Navigation, Path, Control, Avoidance, Motion, BDI, etc. 


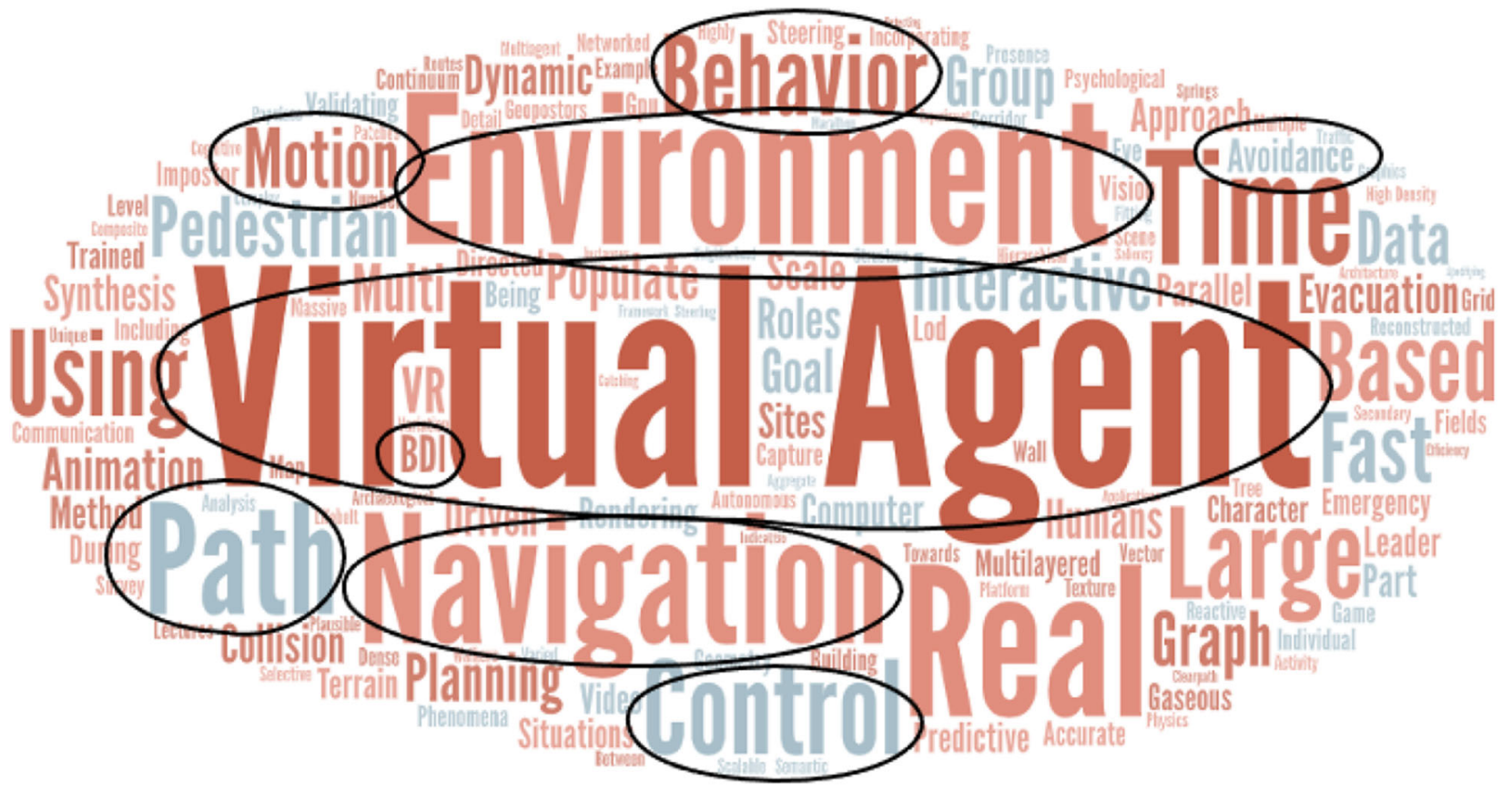

Fig. 5 A set of 146 words compose the cloud of terms from 57 papers published between 2005 and 2009

\subsection{Crowd papers from 2010 to 2014: the consolidation of navigation and collision avoidance techniques, and the beginning of personalities and synthetic vision methods}

Synthetic vision for virtual humans was first introduced by Renault et al. [86] in 1990. In 2010, Ondrej et al. [75], and in 2014, Dutra et al. [28] explored a vision-based approach of collision avoidance between agents into a crowd. Some researchers work with synthetic vision in crowds until nowadays. Other authors proposed techniques for panic situations as Oguz et al. [76], as proposed in the first period of analysis, and data-driven techniques [16,31,113], as firstly described in 2007. In particular, Ju et al. [43] proposed a method that blends existing crowd data to generate a new crowd animation, called morphable crowds.

Indeed, from 2010 to 2014, some important navigation and collision avoidance algorithms were proposed. The first version of the very known Optimal Reciprocal Collision Avoidance (ORCA) method was proposed in 2011 [8], and until now, it is a method very used in crowd domain benchmarks. BioCrowds was proposed by Bicho et al. [11] and claimed to be the first proven free-of-collision algorithm for crowds. The method is based on space subdivision, where agents compete for space with others and move only if there is enough space.

Personalities [26], human relationships [74], and populations with purpose [57] are examples of features used to endow agents into the crowds in this period. Durupinar et al. [26] extend the HiDAC (High-Density Autonomous
Crowds), proposed by Pelechano et al. [78], by providing each agent with a personality model based on the Ocean (openness, conscientiousness, extroversion, agreeableness, and neuroticism) personality model. Each personality trait leads to the automation of low-level behavior parameters. Okaya [74] proposes a human behavior model in evacuation scenarios based on BDI and Helbing's model. Human relationships affect the states of BDI, and altruism forces among agents affect Helbing's computed movements.

Nevertheless, some frameworks and methods to test and compare crowd models have been proposed. Musse et al. [70] propose an approach to compare crowds using 4D histograms having velocities, orientations, and positions of agents in the space. Wolinski et al. [103] presented a framework to evaluate multi-agent crowd simulation algorithms based on real-world observations of crowd movements. Shoulson et al. [93] propose ADAPT, a tool to develop prototyping testbed in crowd scenarios. In addition, SteerPlex, a model to estimate the complexity of crowd simulation scenarios, is proposed by Berseth et al. [9], as its extension SteerFit, which goal is to propose an automatic parameter fitting for steering algorithms [10].

Looking to Fig. 6, we can see the titles of 77 papers selected in this period. We perceive that most of the words are similar to Fig. 5, so in our analysis, we can say that in this period, researchers in crowds seem to consolidate methods for navigation and collision avoidance, steering behaviors, and start other areas such as synthetic vision, for instance. 


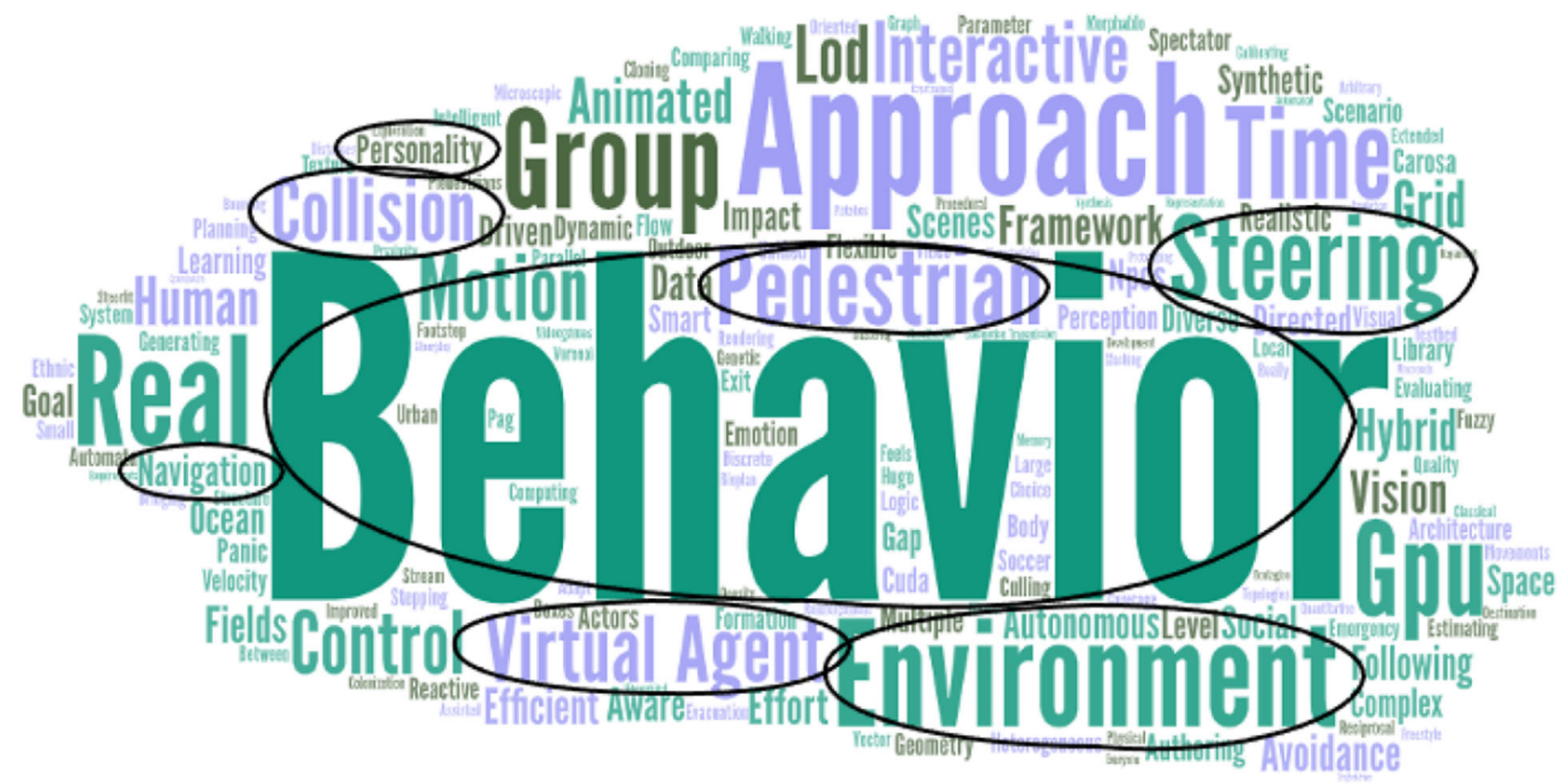

Fig. 6 The 173 words extracted from 77 papers published between 2010 and 2014

\subsection{Crowd papers from 2015 to 2019: the era of learning techniques}

From 2015 to 2019, we keep having papers related to datadriven methods, and a significant number include machine learning and statistical prediction techniques. Authors describe methods to extract real crowds data to describe efficient trajectories and combine with a learned crowdsimulation model [7]. Ahmet et al. [6] propose a data-driven approach for tuning, validating, and optimizing crowd simulations by learning parameters from real-life videos. Liu et al. [60] describe a method to predict crowd dynamics' aggregate characteristics using regression neural networks (NN). Ravichandran et al. [84] model pedestrians as autonomous, learning, and reactive agents employing reinforcement learning (RL). Amirian et al. [1] present a data-driven crowd simulation method that can mimic pedestrians' observed traffic in a given environment learned using generative adversarial networks (GANs). Also, using artificial neural networks, Testa et al. [97] propose a novel approach to estimate the total evacuation time of a given complex environment with an average error of 5\% when compared to evacuation time in a real-life environment. The authors train artificial neural networks (ANNs) to learn the evacuation time of various size rooms, therefore aggregating per-room information, so the full environment can be properly estimated using ANNs.

One topic that is recurrent in all periods is evacuation. Cassol et al. [15] publish a book just on crowd evacuation, showing many applications and real case scenarios. Wong et al. [105] present an algorithm to compute the optimal route for each local region of an environment. The idea is to reduce congestion and maximize the number of evacuees arriving at exits in each time. The system repeats until an optimal state is achieved. Bianco et al. [22] describe a statistical method to provide the crowd motion in a future time based on a specified time. The authors use Pedestrian Dead-Reckoning (PDR) to project future positions $t+K$, based on a time $t$, where $t$ is a specific time, and $K$ is the desired shift in the future time. In addition, the authors considered the complexity of the environment and a statistical distribution to represent, respectively, the impact of obstacles and the other agents in future positions of agents. Mathew [64] propose an inverse approach that is useful both to crowd simulation in virtual environments and urban crowd planning applications. Indeed, the authors propose a way where the crowd impacts the environment using optimization algorithms. Ren et al. [85] combine physics-based simulation methods with datadriven techniques using an optimization-based formulation to simulate tens or hundreds of agents at interactive rates. Results of simulation are compared with real-world datasets in terms of accuracy.

In addition to learning techniques, authoring systems have also been proposed at this period. In particular, Ho et al. [37] propose an authoring system to edit crowd distributions in different time instances, as an extension of the method proposed by Krontiris [50], where the environment is modeled as an influence map based on attractors used to guide crowd simulation activities. In terms of navigation and crowd collision, in 2017, Stüvel et al. [94] present a novel dense crowd simulation method. In real crowds of high density, people maneuvering the crowd need to twist their torso to 
pass between others. Their method does not use the traditional disc-shaped agent, but instead employs capsule-shaped agents, which permit to plan such torso orientations. Contrary to other crowd simulation systems, which often focus on the movement of the entire crowd, the method distinguishes between active agents that try to maneuver through the crowd, and passive agents that have no incentive to move. The authors introduce the concept of a focus point to influence crowd agent orientation.

In this period, we also have more papers in Virtual, Augmented, and Mixed realities [23,41,67]. Moussaïd et al. [67] propose a shared three-dimensional virtual environment as an experimental platform for conducting crowd experiments with real people. Interestingly, they showed that crowds of real human subjects exhibit typical patterns of real crowds, as observed in crowded real-life situations. In addition, some researchers propose papers that evaluate and measure the impact of user's presence, such as $[51,80,108]$. For instance, in [54], authors propose to investigate performance and user experience in Social Virtual Reality (SVR) embodied, immersive, and providing face-to-face encounters. The user study showed positive effects of presence feeling and the possibility of interaction and co-presence.

Furthermore, in this analysis, we included papers in cultural and personality aspects. Favaretto et al. $[27,30]$ introduce a new method to crowd analysis extracting Hofstede dimensions from video sequences, to later be used in crowd simulation [24]. Durupinar et al. [27] propose to parameterize mobs' common properties to create collective behaviors. Their model associates psychological components with individual agents into the crowd providing emergent behaviors. Also, a recurrent subject is the group formation. Here we mention the work of Zhang et al. [111] where authors propose a way to control crowds based on constrained and controlled groups formation. Although there are many methods in crowds contagion nowadays, we mention two of them introduced in this period. Borg et al. [12] proposed an extension of Bosse's model [13] specifically to the crowds' domain. In their work, authors propose to propagate the emotion among agents in the crowd. Basak et al. [5] propose a data-driven approach for tuning crowd simulations with parameters from real-life videos. Authors demonstrate their method in three real-life incidents: a bombing attack, a panic situation on the subway, and a Black Friday rush, where agents' behaviors considered panic contagion.

Finally, analyzing Fig. 7 one can note the importance of data-driven and learning in the word cloud and interactive, experience, VR, AR, contagion, group behavior, emotion words, as discussed in this section.
3 New trends: papers from 2020 and beyond

This section presents some papers from the selected 46 published in this period. Indeed, we included some papers on the crowd domain published in 2021. Certainly, many more papers are going to be published in 2021. For the papers cited in this work, we searched in Google scholar with keywords crowd and simulation. We selected the papers that focus on crowd simulation and papers that were peer-reviewed in relevant forums.

Figure 8 shows the word cloud generated based on selected titles. It is interesting to see that the most used term, after crowd simulation, discarded in all periods, is again pedestrian (as the period before 2000), followed by Virtual Human (as in the period 2000-2004) and not Virtual Agent as the lasts periods. It may indicate the community tendency to simulate individuals with more human characteristics, as emotion [100,107], natural language [61], among others. Besides, authors propose methods to individualize agents into the crowd, as for instance providing door and doorway etiquette [38], distracted agents [49], providing agents which motion is impacted by fluid forces [91] and obeying social distance in pandemic situations [44]. We also saw papers mixing crowds in VR environments, as proposed by [102], where authors study the effects on users during interaction with a virtual human crowd in an immersive virtual reality environment or still exploring tactile feedback during immersive walking in VR applications [48].

In this period, we found some papers exploring artificial neural networks to predict crowd emotions [100] and to integrate data-driven walking behaviors [47]. Crowd analysis is still present in papers in 2020. Li et al. [58] propose quantifying and detecting collective motion through a multi-stage clustering strategy and a method to provide crowd anomaly detection and localization using histogram of magnitude and momentum [4]. Regarding crowds and environments: Sun et al. [95] propose to predict crowd flows using graph convolutional networks. Zhou et al. [115] present a case study of Beijing Subway stations. This paper proposes a modified social force model to investigate crowd evacuation dynamics taking into account the influence of emergency signs.

High-dense crowds and evacuation scenarios is kept being studied in 2020. Firstly, the study of the impact of panic on crowd movement is based on information entropy [114], and the investigation of the evacuation path optimization (EPO) problem using ant colony inspiration [39]. Finally, high abstractions of crowds have been proposed as BioClouds and Legion models [20,21], where authors want to simulate huge big crowds, e.g., a million of people, however without simulating each individual, but keeping the global crowd properties and the possibility to zoom in individual behaviors. 


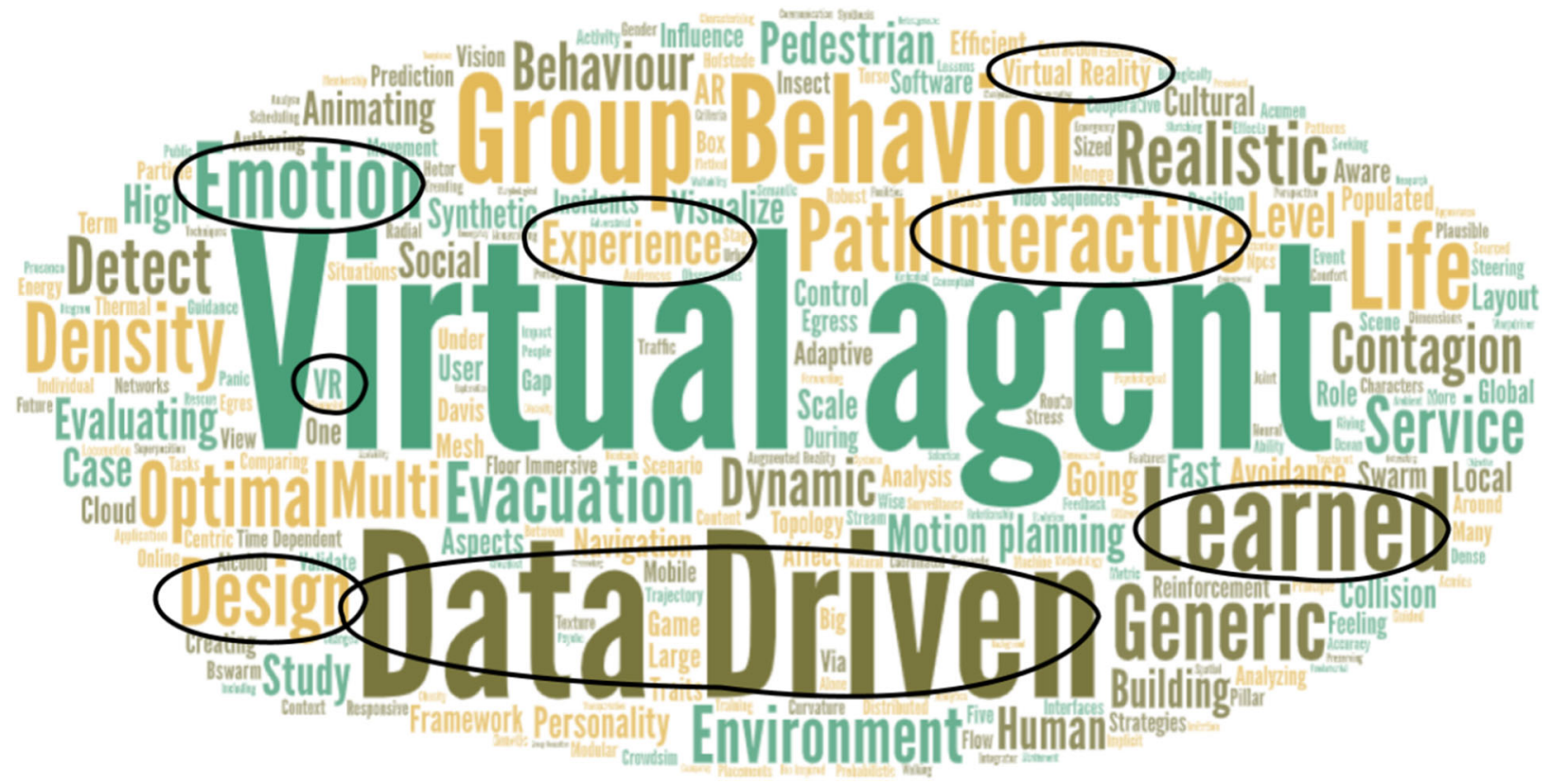

Fig. 7276 words extracted from 106 papers published between 2015 and 2019

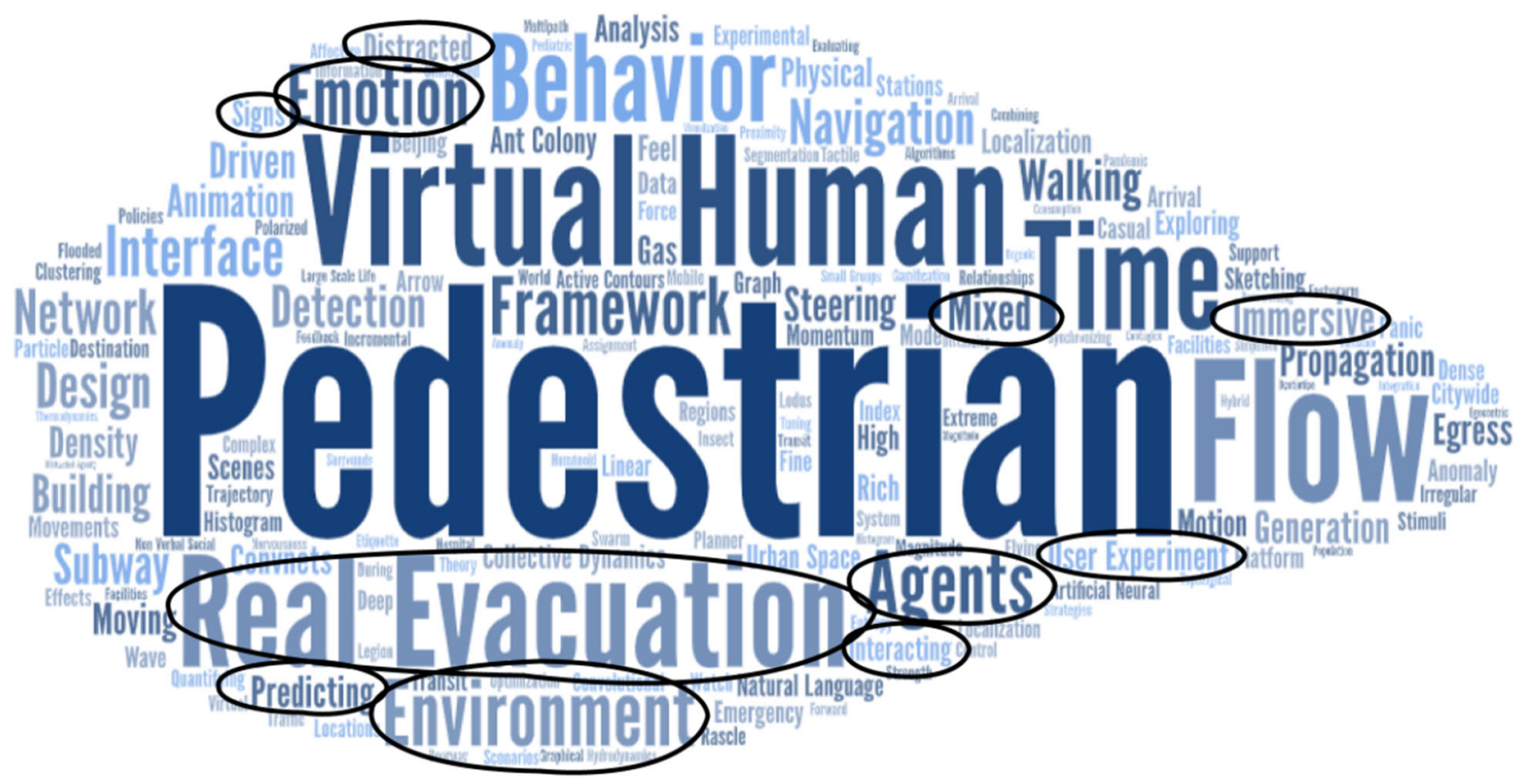

Fig. 8 The 173 terms extracted from 46 papers published in 2020 and 2021

In 2021, some methods have already been proposed to assist the mobility of people in scenarios of evacuation of buildings, dangerous situations such as a terrorist attack, strategies to find optimal routes, the impact of groups of agents on the efficiency of the crowd, and even to simulate human mobility during the COVID-19 situation. Here are some examples: Mirahadi and McCabe [65] propose a real-time model for building evacuation scenarios based on Dijkstra's very know algorithm [25]. Li et al. [59] present a rule-based model based on an extended cellular automata model considering groups of agents to study Pediatric Hospital evacuation. Xie et al. [106] propose a model that accounts for social groups, based on a social force framework, to investigate evacuation dynamics in pedestrian crowds impacted by groups of agents. Other authors have also proposed approaches with group behaviors in recent years, for example $[46,66]$. Zhang et al. [112] investigate an optimal guidance strategy for large-scale crowd evacuations, increasing the 


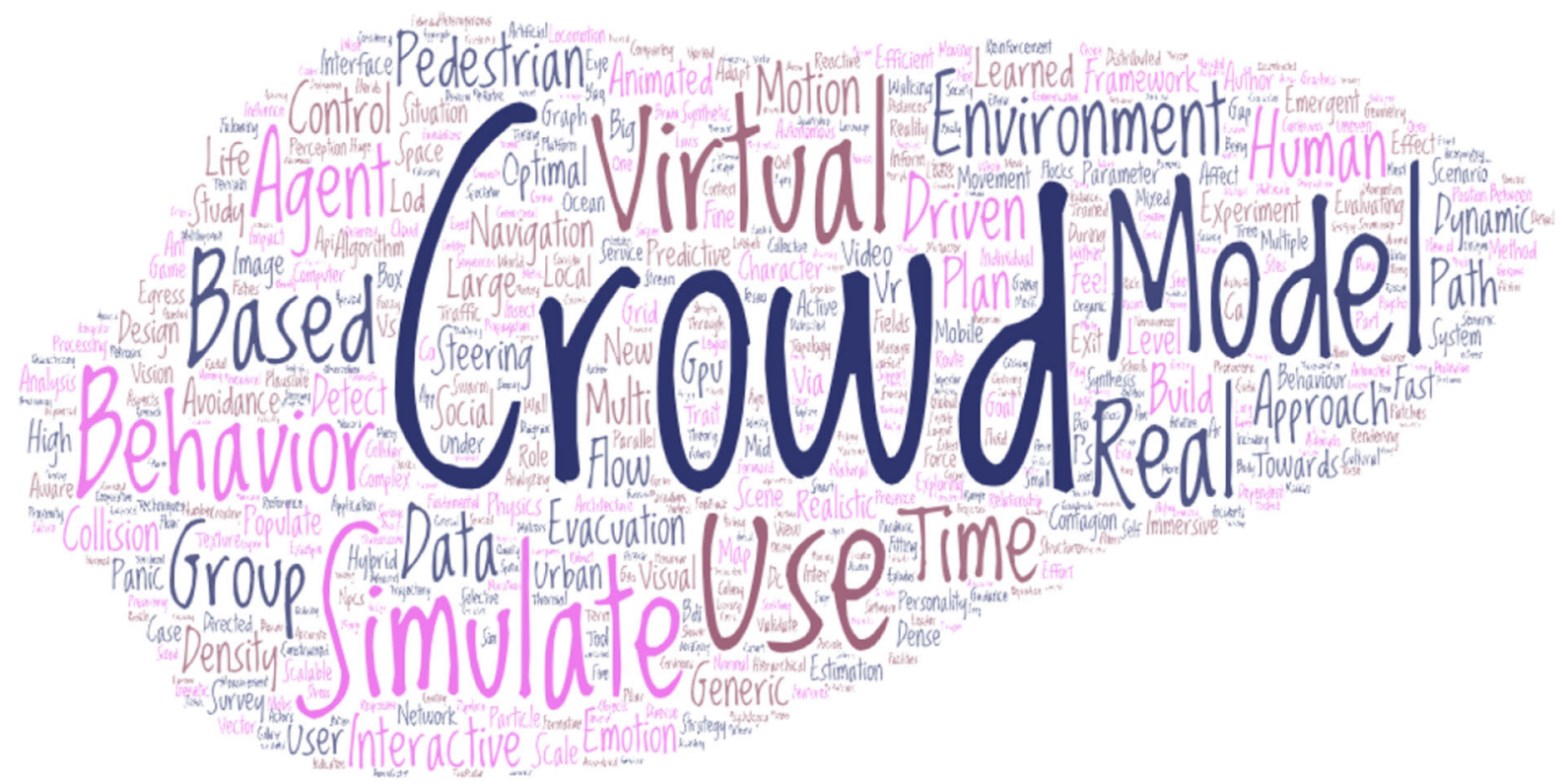

Fig. 9600 terms extracted from 319 papers published since 1987 in crowd simulation

computational efficiency. Charrier et al. [17] propose an approach using the well-known Helbing's model [35] to include a nervousness propagation mechanism, and then the authors study its impact on a classical simulation setup. Furthermore, finally, Shi et al. [92] propose a work which goal is to verify the applicability of the Social Force Model (SFM), embedded in Viswalk software, to reproduce the effect of egress flow under normal and emergency conditions. As can be observed, many papers have been proposed in the context of evacuation and crowd mobility, as the example of Orallo and Martinez [36]. The authors study models for evaluating the temporal and spatial risk of transmission of the COVID19 as a function of humans mobility.

\section{Final discussion}

This paper presented a history of crowd simulation focusing mainly on how the many techniques that were proposed in the past were organized over time. For instance, it is interesting to show the evolution of used terms, as Pedestrian (until 1999), Virtual Human (2000-2004), Virtual Agent (2005-2009), Virtual Agent and Pedestrian (2010-2014), again Virtual Agent (2015-2019), and finally, Pedestrian and Virtual Human, that appeared again in 2020 and 2021. In terms of research that keep being proposed since the early years, we can cite evacuation models, crowd, group, and individual behaviors, and all types of navigation methodologies. The recent tendencies that seem to be strong nowadays (and probably in next years) are: data-driven techniques, the usage of machine learning algorithms applied to crowds, the

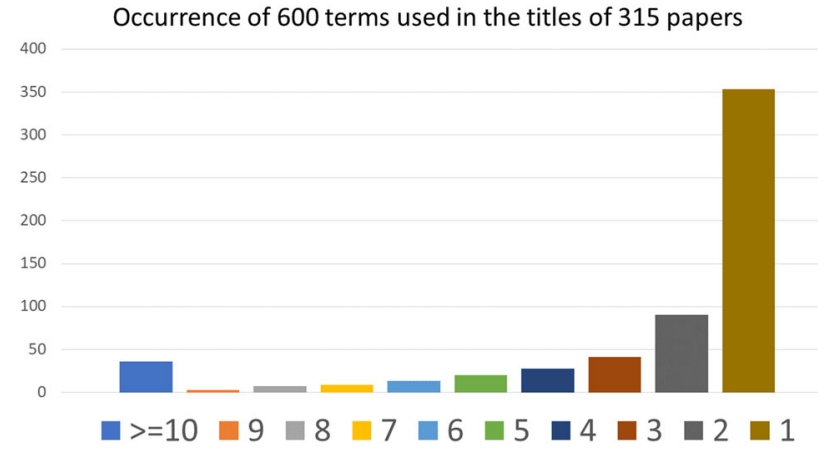

Fig. 10 Histogram of occurrences of 600 terms extracted from 319 papers published since 1987 in crowd simulation

specialization of individual behaviors to be more "human" and "intelligent," and recent papers show intense energy focused on evacuation applications. In the latter case, we believe that more evacuation techniques will be proposed trying to include other methods such as individual behaviors and machine learning methods, at the same time. So, we believe the most relevant application is still the evacuation of scenarios in the crowd area. Figure 9 shows the word cloud generated using 600 different terms in the titles of papers in the crowd domain since 1987. Thirty-six of these words, shown in Table 1, were used more than ten times in the titles. We highlighted some words in the table as crowd (225 occurrences), Agent (32), Pedestrian (26), Human (21), and Evacuation (20). Also, Fig. 10 shows the histogram of terms repetition in the titles.

There is still a long way to go to achieve very realistic crowd simulations, as expected in real life. While crowd 
Table 1 The table presents the terms used more or equal 10 occurrences in the titles of 319 papers, since 1987

\begin{tabular}{llllll}
\hline Word & Count & Word & Count & Word & Count \\
\hline Crowd & $\mathbf{2 2 5}$ & Simulate & 153 & Model & 59 \\
Virtual & 55 & Behavior & $\mathbf{4 4}$ & Use & 42 \\
Based & 41 & Agent & $\mathbf{3 2}$ & Environment & $\mathbf{2 9}$ \\
Real & 29 & Pedestrian & $\mathbf{2 6}$ & Group & $\mathbf{2 6}$ \\
Time & 24 & Human & $\mathbf{2 1}$ & Interactive & 21 \\
Approach & 21 & Evacuation & $\mathbf{2 0}$ & Driven & $\mathbf{1 8}$ \\
Navigation & $\mathbf{1 7}$ & Data & 17 & Motion & 15 \\
Dynamic & 15 & Animated & 15 & Steering & 13 \\
Density & 13 & Control & 13 & Generic & 12 \\
Plan & 12 & Emotion & $\mathbf{1 2}$ & Collision & 11 \\
Learned & 11 & Framework & 10 & Optimal & 10 \\
Large & 10 & Build & 10 & Populate & 10 \\
\hline
\end{tabular}

simulation is generally seen as the process of simulating the movement of many entities or characters, in reality, huge crowds generally do not walk, e.g., 90\% of crowd images on Google Images shows static crowds. Nevertheless, even when crowds move, they can run or even swim in a crowded pool. Most path planning algorithms for walking will not work for swimming. People in a city generally walk and stop only to watch events or chat with people. Many people in public parks would sit down on the grass or on public seats. We should see more children, babies, old people, and disabled people in terms of crowd appearance. We should be able to mix people with cars, bicycles, etc. Currently, individuals in crowds can carry accessories, what we do not see is crowds manipulating objects, opening doors, eating, moving objects from one place to another, exchanging objects. Other examples of scenes we have not see until now in crowd simulations are large restaurants, crowded buses, trains, etc.

Most simulated crowds are composed of people with a goal, but in real life, on a Sunday afternoon, many people wander without specific goals. We ultimately need to introduce natural motivations to simulate more complex and realistic situations. For example, in an airport, people should not just check-in, go to the security then the gate, as in most simulations. They should be able to go to restaurants, cafes, shops, toilets, maybe meet someone, according to their internal motivations. Such models exist, but the problem is that it will be extremely CPU intensive to introduce them in simulations, having diversity and realism while being as autonomous as possible, avoiding creating extra work for designers. Indeed, the control is still an issue in crowd simulation because, while we want autonomous and emergent crowds evolving in a virtual environment endowed with realistic motivations to be credible, we also want to interact with the individuals and make a new narrative or play a game. Much have been studied in the crowd area, as this paper showed, but certainly, much more remains to be done.
For new perspectives, the following are some possibilities, among many others:

- To improve the crowd properties and realism in macroscopic behaviors, using machine learning techniques, as it has been made in recent papers. The goal is to have more intelligent crowds at the macrolevel;

- To increase the realism of individual behaviors, as personalities, cultural aspects, and emotions, providing more intelligent and specific individuals, even including facial and posture motion;

- The last two items must work together, providing a method to zoom-in and zoom-out in crowd simulation to enable the visualization of details at the desired level. Also, the individuals should be part of the collective behavior in a synchronized and coherent way;

- To provide realistic crowds in VR and AR applications, including macro- and microbehaviors, facial, body, and cloth animation. Users should be able to select the level of details they want to control, and finally

- New methodologies in highly dense crowds, including all micro-level behaviors and evacuation applications, integrated with computer vision techniques.

Since the seminal paper of Reynolds [88], much has been done in crowd simulation research. This paper presents a view of the history of proposed crowd simulation methods and the main achievements. A dataset containing more than 300 papers ${ }^{10}$ published in specific conferences and journals is discussed in depth. The crowd domain is one area where many researchers have worked for many years, and we are still far from having a model where we can firmly believe in crowd behaviors at micro- and macrolevels. It indeed well resumes the challenge of the area. The future points

\footnotetext{
$\overline{10}$ The list of papers is available upon request.
} 
to integration with machine learning methods and hardware development, which will undoubtedly bring new achievements to this area.

Funding Soraia R. Musse was funded by $\mathrm{CNPq}$ (Grant number: 305084/2016-0) and FAPERGS (Grant number: 20/2551-0000280-2), and Vinicius Cassol was funded by FAPERGS (Grant number: 20/25510000280-2).

\section{Declarations}

Conflict of interest The authors declare that they have no conflict of interest.

\section{References}

1. Amirian, J., van Toll, W., Hayet, J.B., Pettré, J.: Data-driven crowd simulation with generative adversarial networks. In: Proceedings of the 32nd International Conference on Computer Animation and Social Agents, CASA '19, p. 7-10. Association for Computing Machinery, New York, NY, USA (2019). https://doi.org/10.1145/ 3328756.3328769

2. Anderson, M., McDaniel, E., Chenney, S.: Constrained animation of flocks. In: Proc. ACM SIGGRAPH/Eurographics Symposium on Computer Animation (SCA'03), pp. 286-297 (2003)

3. Azahar, M.A.B.M., Sunar, M.S., Daman, D., Bade, A.: Survey on real-time crowds simulation. In: Pan, Z., Zhang, X., El Rhalibi, A., Woo, W., Li, Y. (eds.) Technologies for E-Learning and Digital Entertainment, pp. 573-580. Springer, Berlin Heidelberg (2008)

4. Bansod, S., Nandedkar, A.: Crowd anomaly detection and localization using histogram of magnitude and momentum. Visual Comput. 36, 609-620 (2020). https://doi.org/10.1007/s00371019-01647-0

5. Başak, A., Gudukbay, U., Durupinar, F.: Using real life incidents for realistic virtual crowds with data-driven emotion contagion. Comput. Graph. (2018). https://doi.org/10.1016/j.cag.2018.02. 004

6. Başak, A.E., Güdükbay, U., Durupınar, F.: Using real life incidents for creating realistic virtual crowds with data-driven emotion contagion. Comput. Graph. 72, 70-81 (2018). https://doi.org/10. 1016/j.cag.2018.02.004

7. Bera, A., Kim, S., Manocha, D.: Efficient trajectory extraction and parameter learning for data-driven crowd simulation. In: Proceedings of Graphics Interface 2015, GI 2015, pp. 65-72. Canadian Human-Computer Communications Society, Toronto, Ontario, Canada (2015). https://doi.org/10.20380/GI2015.09

8. van den Berg, J., Guy, S., Lin, M., Manocha, D.: Reciprocal nbody collision avoidance. Springer Tracts Adv. Robot. 70, 3-19 (2011)

9. Berseth, G., Kapadia, M., Faloutsos, P.: Steerplex: Estimating scenario complexity for simulated crowds. In: International Conference of Motion on Games, pp. 45:67-45:76. ACM, New York, NY, USA (2013)

10. Berseth, G., Kapadia, M., Haworth, B., Faloutsos, P.: Steerfit: Automated parameter fitting for steering algorithms. In: Proceedings of the ACM SIGGRAPH/Eurographics Symposium on Computer Animation, SCA '14, p. 113-122. Eurographics Association, Goslar, DEU (2015)

11. Bicho, A.L., Rodrigues, R.A., Musse, S.R., Jung, C.R., Paravisi, M., Magalh, L.P.: Simulating crowds based on a space colonization algorithm. Comput. Graph. 36(2), 70-79 (2012)
12. Borges Fortes Neto, A., Pelachaud, C., Musse, S.R.: Giving emotional contagion ability to virtual agents in crowds. In: Beskow, J., Peters, C., Castellano, G., O’Sullivan, C., Leite, I., Kopp, S. (eds.) Intelligent Virtual Agents, pp. 63-72. Springer, Cham (2017)

13. Bosse, T., Hoogendoorn, M., Klein, M.C.A., Treur, J., van der Wal, C.N.: Agent-based analysis of patterns in crowd behaviour involving contagion of mental states. In: K.G. Mehrotra, C.K. Mohan, J.C. Oh, P.K. Varshney, M. Ali (eds.) Modern Approaches in Applied Intelligence: 24th International Conference on Industrial Engineering and Other Applications of Applied Intelligent Systems, IEA/AIE 2011, Syracuse, NY, USA, June 28 - July 1, 2011, Proceedings, Part II, p. 677. Springer Berlin Heidelberg (2011)

14. Braun, A., Musse, S.R., Oliveira, L.P.L.d., Bodmann, B.E.J.: Modeling individual behaviors in crowd simulation. In: International Conference on Computer Animation and Social Agents (CASA), p. 143 (2003)

15. Cassol, V.J., Musse, S.R., Jung, C.R., Badler, N.I.: Simulating Crowds in Egress Scenarios. Springer, London (2017)

16. Charalambous, P., Chrysanthou, Y.: Learning crowd steering behaviors from examples. In: Boulic, R., Chrysanthou, Y., Komura, T. (eds.) Motion in Games, p. 35. Springer, Berlin Heidelberg (2010)

17. Charrier, R., Philippe, J., Bertelle, C.: Study of a model of nervousness propagation within virtual crowds. In: Prendinger, H., Lester, J., Ishizuka, M. (eds.) Complex Systems, Smart Territories and Mobility, pp. 187-200. Springer, Cham (2021). https:// doi.org/10.1007/978-3-030-59302-5_10

18. Cho, K., Iketani, N., Kikuchi, M., Nishimura, K., Hayashi, H., Hattori, M.: Bdi model-based crowd simulation. In: Prendinger, H., Lester, J., Ishizuka, M. (eds.) Intelligent Virtual Agents, pp. 364-371. Springer, Berlin Heidelberg (2008)

19. Courty, N., Corpetti, T.: Crowd motion capture. Comput. Animat. Virtual Worlds 18(4-5), 361-370 (2007)

20. da Silva Antonitsch, A., Musse, S.R., de Figueiredo, L.H.: Towards a legion of virtual humans: Steering behaviors and organic visualization. In: 2020 33rd SIBGRAPI Conference on Graphics, Patterns and Images (SIBGRAPI), pp. 31-38 (2020). https://doi.org/10.1109/SIBGRAPI51738.2020.00013

21. Da Silva Antonitsch, A., Schaffer, D.H.M., Rockenbach, G.W., Knob, P., Musse, S.R.: Bioclouds: a multi-level model to simulate and visualize large crowds. In: Gavrilova, M., Chang, J., Thalmann, N.M., Hitzer, E., Ishikawa, H. (eds.) Advances in Computer Graphics. Springer International Publishing, Cham (2019)

22. Dal Bianco, C.M., Braun, A., Brasil, J., Musse, S.R.: Preserving the motion features in nonavoiding collision crowds. Comput. Entertan. 15(3), 1-15 (2017)

23. Dickinson, P., Gerling, K., Hicks, K., Murray, J., Shearer, J., Greenwood, J.: Virtual reality crowd simulation: effects of agent density on user experience and behaviour. Virtual Reality (2019). https://doi.org/10.1007/s10055-018-0365-0

24. Dihl, L., Testa, E.S., Knob, P., da Silva, G.L.B., Favaretto, R.M., de Alcântara, M.F., Musse, S.R.: Generating cultural characters based on hofstede dimensions. In: 2017 IEEE Virtual Humans and Crowds for Immersive Environments (VHCIE), pp. 1-5 (2017). https://doi.org/10.1109/VHCIE.2017.7935621

25. Dijkstra, E.W.: A note on two problems in connexion with graphs. Numerische Mathematik 1(1), 269-271 (1959). https://doi.org/ 10.1007/BF01386390

26. Durupinar, F., Pelechano, N., Allbeck, J., Güdükbay, U., Badler, N.I.: How the ocean personality model affects the perception of crowds. IEEE Comput. Graph. Appl. 31(3), 22-31 (2011). https:// doi.org/10.1109/MCG.2009.105

27. Durupınar, F., Güdükbay, U., Aman, A., Badler, N.I.: Psychological parameters for crowd simulation: from audiences to mobs. 
IEEE Trans. Visual. Comput. Graph. 22(9), 2145-2159 (2016). https://doi.org/10.1109/TVCG.2015.2501801

28. Dutra, T., Priem, G., Cavalcante-Neto, J., Vidal, C., Pettre, J.: Synthetic vision-based crowd simulation: Reactive vs. reactive planning approaches. In: Proceedings of the 27th Conference on Computer Animation and Social Agents, pp. 1-4 (2014)

29. Farenc, N., Raupp Musse, S., Schweiss, E., Kallmann, M., Aune, O., Boulic, R., Thalmann, D.: A paradigm for controlling virtual humans in urban environment simulations. Appl. Art. Intell. J. Special Issue Intell. Virtual Environ. 14(1), 69-91 (2000)

30. Favaretto, R., Dihl, L., Barreto, R., Musse, S.R.: Using group behaviors to detect hofstede cultural dimensions. In: IEEE International Conference on Image Processing (ICIP) (2016)

31. Flagg, M., Rehg, J.M.: Video-based crowd synthesis. IEEE Trans. Visual. Comput. Graph. 19(11), 1935-1947 (2013). https://doi. org/10.1109/TVCG.2012.317

32. Geraerts, R., Kamphuis, A., Karamouzas, I., Overmars, M.: Using the corridor map method for path planning for a large number of characters. In: Egges, A., Kamphuis, A., Overmars, M. (eds.) Motion in Games, pp. 11-22. Springer, Berlin Heidelberg (2008)

33. Grillon, H., Thalmann, D.: Simulating gaze attention behaviors for crowds. Comput. Animat. Virtual Worlds (2009). https://doi. org/10.1002/cav.293

34. Helbing, D., Farkas, I., Vicsek, T.: Simulating dynamical features of escape panic (2000). http://www.nature.com/nature/journal/ v407/n6803/

35. Helbing, D., Molnar, P.: Social force model for pedestrian dynamics. Phys. Rev. E 51, 42-82 (1995)

36. Hernández-Orallo, E., Armero-Martánez, A.: How human mobility models can help to deal with covid-19. Electronics 10(1), 33 (2021). https://doi.org/10.3390/electronics10010033. https:// www.mdpi.com/2079-9292/10/1/33

37. Ho, R.C., Wong, S.K., Chou, Y.H., Lin, G.W., Tsai, T.Y., Huang, P.H., Wang, Y.S., Yeh, I.C.: An authoring framework for time dependent crowd simulation. In: SIGGRAPH ASIA 2016 Posters, SA'16. Association for Computing Machinery, New York, NY, USA (2016). https://doi.org/10.1145/3005274.3005278

38. Huang, W., Terzopoulos, D.: Door and doorway etiquette for virtual humans. IEEE Trans. Visual. Comput. Graph. 26(3), 15021517 (2020). https://doi.org/10.1109/TVCG.2018.2874050

39. Huang, Z.M., Chen, W.N., Li, Q., Luo, X.N., Yuan, H.Q., Zhang, J.: Ant colony evacuation planner: An ant colony system with incremental flow assignment for multipath crowd evacuation. IEEE Trans Cybern, pp. 1-14 (2020). https://doi.org/10.1109/ TCYB.2020.3013271

40. H.Y., S., Shivakumar, G., Mohana, H.S.: Crowd behavior analysis: A survey. In: 2017 International Conference on Recent Advances in Electronics and Communication Technology (ICRAECT), pp. 169-178 (2017). https://doi.org/10.1109/ICRAECT.2017.66

41. Hürst, W., Geraerts, R.: Augmented and virtual reality interfaces for crowd simulation software-a position statement for research on use-case-dependent interaction. In: 2019 IEEE Virtual Humans and Crowds for Immersive Environments (VHCIE), pp. 1-3 (2019). https://doi.org/10.1109/VHCIE.2019.8714733

42. Ijaz, K., Sohail, S., Hashish, S.: A survey of latest approaches for crowd simulation and modeling using hybrid techniques. In: Proceedings of the 2015 17th UKSIM-AMSS International Conference on Modelling and Simulation (2015)

43. Ju, E., Choi, M.G., Park, M., Lee, J., Lee, K.H., Takahashi, S.: Morphable crowds. ACM Trans. Graph. 29, 6 (2010). https://doi. org/10.1145/1882261.1866162

44. Kapadia;, U.L.M.Z..F.: A social distancing index: Evaluating navigational policies on human proximity using crowd simulations. In: ACM SIGGRAPH Conference - Motion in Games, p. 1 (2020)

45. Karamouzas, I., Heil, P., van Beek, P., Overmars, M.H.: A predictive collision avoidance model for pedestrian simulation. In:
Egges, A., Geraerts, R., Overmars, M. (eds.) Motion in Games, pp. 41-52. Springer, Berlin Heidelberg (2009)

46. Karamouzas, I., Overmars, M.: Simulating and evaluating the local behavior of small pedestrian groups. IEEE Trans. Visual. Comput. Graph. 18(3), 394-406 (2012). https://doi.org/10.1109/ TVCG.2011.133

47. Kielar, P., Borrmann, A.: An artificial neural network framework for pedestrian walking behavior modeling and simulation. Collect. Dynam. 5, 290-298 (2020)

48. Koilias, A., Mousas, C., Anagnostopoulos, C.N.: I feel a moving crowd surrounds me: exploring tactile feedback during immersive walking in a virtual crowd. Comput. Anim. Virtual Worlds 31(4 5), 1963 (2020). https://doi.org/10.1002/cav.1963

49. Kremer, M., Haworth, B., Kapadia, M., Faloutsos, P.: Modelling distracted agents in crowd simulations. Visual Comput. 37(1), 1432-2315 (2021). https://doi.org/10.1007/s00371-020-019694

50. Krontiris, A., Bekris, K.E., Kapadia, M.: Acumen: Activitycentric crowd authoring using influence maps. In: 29th International Conference on Computer Animation and Social Agents (CASA) Geneva, Switzerland (2016)

51. Kyriakou, M., Chrysanthou, Y.: How responsiveness, group membership and gender affect the feeling of presence in immersive virtual environments populated with virtual crowds. In: Proceedings of the 11th Annual International Conference on Motion, Interaction, and Games, MIG '18, Association for Computing Machinery, New York, NY, USA (2018). https://doi.org/10.1145/ 3274247.3274509

52. Lai, Y.C., Chenney, S., Fan, S.: Group motion graphs. In: Proceedings of the 2005 ACM SIGGRAPH/Eurographics Symposium on Computer Animation, SCA'05, p. 281-290. Association for Computing Machinery, New York, NY, USA (2005). https://doi.org/ 10.1145/1073368.1073409

53. Lamarche, F., Donikian, S.: Crowd of virtual humans: a new approach for real time navigation in complex and structured environments. Comput. Graphics Forum 23(3), 509-518 (2004). https://doi.org/10.1111/j.1467-8659.2004.00782.x

54. Latoschik, M.E., Kern, F., Stauffert, J.P., Bartl, A., Botsch, M., Lugrin, J.L.: Not alone here?! scalability and user experience of embodied ambient crowds in distributed social virtual reality. IEEE Trans. Visual. Comput. Graph. 25(5), 2134-2144 (2019). https://doi.org/10.1109/TVCG.2019.2899250

55. Lee, K.H., Choi, M.G., Hong, Q., Lee, J.: Group behavior from video: A data-driven approach to crowd simulation. In: Proceedings of the 2007 ACM SIGGRAPH/Eurographics Symposium on Computer Animation, SCA ’07, pp. 109-118. Eurographics Association, Aire-la-Ville, Switzerland, Switzerland (2007). http://dl. acm.org/citation.cfm?id=1272690.1272706

56. Lerner, A., Chrysanthou, Y., Lischinski, D.: Crowds by example. Comput. Graph. Forum 26(3), 655-664 (2007). https://doi.org/ 10.1111/j.1467-8659.2007.01089.x

57. Li, W., Allbeck, J.M.: Populations with purpose. In: Allbeck, J.M., Faloutsos, P. (eds.) Motion in Games, pp. 132-143. Springer, Berlin Heidelberg (2011)

58. Li, X., Chen, M., Wang, Q.: Quantifying and detecting collective motion in crowd scenes. IEEE Trans. Image Process. 29, 55715583 (2020). https://doi.org/10.1109/TIP.2020.2985284

59. Li, Y., Lu, C., Jin, J.: Simulation of a pediatric hospital in evacuation considering groups. Simul. Modell. Prac. Theory (2021). https://doi.org/10.1016/j.simpat.2020.102150

60. Liu, W., Pavlovic, V., Hu, K., Faloutsos, P., Yoon, S., Kapadia, M.: Characterizing the relationship between environment layout and crowd movement using machine learning. In: Proceedings of the Tenth International Conference on Motion in Games, MIG '17, Association for Computing Machinery, New York, NY, USA (2017). https://doi.org/10.1145/3136457.3136474 
61. Liu, W.Y., Wong, S.K., Chen, C.Y.: A natural language interface with casual users for crowd animation. Comput. Animat. Virtual Worlds 31(4-5), 1965 (2020). https://doi.org/10.1002/cav.1965

62. Loscos, C., Marchal, D., A.Meyer: Intuitive crowd behavior in dense urban environments using local laws. In: Proc. Theory and Practice of Computer Graphics (TPCG'03), pp. - (2003)

63. Maim, J., Yersin, B., Thalmann, D.: Unique character instances for crowds. IEEE Comput. Graph. Appl. 29(6), 82-90 (2009). https://doi.org/10.1109/MCG.2009.129

64. Mathew, C.D.T., Knob, P.R., Musse, S.R., Aliaga, D.G.: Urban walkability design using virtual population simulation. Comput. Graph. Forum 38(1), 455-469 (2019). https://doi.org/10.1111/ cgf. 13585

65. Mirahadi, F., McCabe, B.Y.: Evacusafe: a real-time model for building evacuation based on dijkstra's algorithm. J. Build. Eng. (2021). https://doi.org/10.1016/j.jobe.2020.101687

66. Moussaïd, M., Perozo, N., Garnier, S., Helbing, D., Theraulaz, G.: The walking behaviour of pedestrian social groups and its impact on crowd dynamics. Plos One 5(4), 1-7 (2010). https://doi.org/ 10.1371/journal.pone.0010047

67. Moussaïd, M., Kapadia, M., Thrash, T., Sumner, R.W., Gross, M., Helbing, D., Hölscher, C.: Crowd behaviour during high-stress evacuations in an immersive virtual environment. J. Royal Soc. Interf. (2016). https://doi.org/10.1098/rsif.2016.0414

68. Musse, S., Jung, C., Jacques, J.C.S., Braun, A.: Using computer vision to simulate the motion of virtual agents: research articles. Comput. Animat. Virtual Worlds 18(2), 83-93 (2007)

69. Musse, S., Thalmann, D.: A model of human crowd behavior: Group inter-relationship and collision detection analysis, pp. 3951 (1997)

70. Musse, S.R., Cassol, V.J., Jung, C.R.: Towards a quantitative approach for comparing crowds. Comput. Animat. Virtual Worlds 23(1), 49-57 (2012)

71. Musse, S.R., Thalmann, D.: Hierarchical model for real time simulation of virtual human crowds. IEEE Transac. Visual. Comput. Graph. 7(2), 152-164 (2001)

72. Narain, R., Golas, A., Curtis, S., Lin, M.C.: Aggregate dynamics for dense crowd simulation. In: ACM SIGGRAPH Asia 2009 Papers, pp. 122:1-122:8 (2009)

73. Nasir, F.M., Sunar, M.S.: A survey on simulating real-time crowd simulation. In: 2015 4th International Conference on Interactive Digital Media (ICIDM), pp. 1-5 (2015). https://doi.org/10.1109/ IDM.2015.7516327

74. Okaya, M., Takahashi, T.: Human relationship modeling in agentbased crowd evacuation simulation. In: D. Kinny, J.Y.j. Hsu, G. Governatori, A.K. Ghose (eds.) Agents in Principle, Agents in Practice. Springer Berlin Heidelberg (2011)

75. Ondřej, J., Pettré, J., Olivier, A.H., Donikian, S.: A syntheticvision based steering approach for crowd simulation. ACM Trans. Graph. (2010). https://doi.org/10.1145/1778765.1778860

76. Oğuz, O., Akaydın, A., Yılmaz, T., Güdükbay, U.: Emergency crowd simulation for outdoor environments. Comput. Graph. 34(2), 136-144 (2010). https://doi.org/10.1016/j.cag.2009.12. 004

77. Paris, S., Gerdelan, A., O'Sullivan, C.: CA-LOD: collision avoidance level of detail for scalable, controllable crowds. In: Motion in Games, Second International Workshop, MIG, 2009, pp. 1328. Zeist,The Netherlands, November 21-24, 2009. Proceedings ((2009))

78. Pelechano, N., Allbeck, J.M., Badler, N.I.: Controlling individual agents in high-density crowd simulation. In: Proceedings of the 2007 ACM SIGGRAPH/Eurographics Symposium on Computer Animation, SCA '07, p. 99-108. Eurographics Association, Goslar, DEU (2007)
79. Pelechano, N., Allbeck, J.M., Badler, N.I.: Virtual crowds: methods, simulation, and control. Synth. Lect. Comput. Graph. Animat. 3(1), 1-176 (2008)

80. Pelechano, N., Allbecky, J.M.: Feeling crowded yet?: crowd simulations for vr. In: 2016 IEEE Virtual Humans and Crowds for Immersive Environments (VHCIE), pp. 17-21 (2016). https://doi. org/10.1109/VHCIE.2016.7563568

81. Pelechano, N., Badler, N.I.: Modeling crowd and trained leader behavior during building evacuation. IEEE Comput. Graph. Appl. 26(6), 80-86 (2006). https://doi.org/10.1109/MCG.2006.133

82. Pelechano, N., O'brien, K., Silverman, B., Badler, N.: Crowd simulation incorporating agent psychological models, roles and communication. In: V-Crowds, vol. 43, pp. 21-30 (2005)

83. Pettre, J., Ciechomski, P.H., Maim, J., Yersin, B., Laumond, J.P., Thalmann, D.: Real-time navigating crowds: scalable simulation and rendering. Comput. Animat. Virtual Worlds 17(3-4), 445455 (2006)

84. Ravichandran, N.B., Yang, F., Peters, C., Lansner, A., Herman, P.: Pedestrian simulation as multi-objective reinforcement learning. In: Proceedings of the 18th International Conference on Intelligent Virtual Agents, IVA '18, p. 307-312. Association for Computing Machinery, New York, NY, USA (2018). https://doi.org/10.1145/ 3267851.3267914

85. Ren, J., Xiang, W., Xiao, Y., Yang, R., Manocha, D., Jin, X.: Hetersim: Heterogeneous multi-agent systems simulation by interactive data-driven optimization. IEEE Transactions on Visualization and Computer Graphics p. 1 (2019). https://doi.org/10.1109/TVCG. 2019.2946769

86. Renault, O., Thalmann, N.M., Thalmann, D.: A vision-based approach to behavioural animation. J. Visual. Comput. Anim. 1(1), 18-21 (1990). https://doi.org/10.1002/vis.4340010106

87. Reynolds, C.: Steering behaviors for autonomous characters. In: Proceedings of Game Developers Conference 1999. Miller Freeman Game Group (1999)

88. Reynolds, C.W.: Flocks, herds and schools: A distributed behavioral model. In: Proceedings of the 14th Annual Conference on Computer Graphics and Interactive Techniques, SIGGRAPH '87, pp. 25-34 (1987)

89. Rodrigues, R.A., de Lima Bicho, A., Paravisi, M., Jung, C.R., Magalhães, L.P., Musse, S.R.: Tree paths: A new model for steering behaviors. In: Ruttkay, Z., Kipp, M., Nijholt, A., Vilhjalmsson H.H. (eds.) Intelligent Virtual Agents, pp. 358-371. Springer Berlin, Heidelberg (2009)

90. Saleh, S.A.M., Suandi, S.A., Ibrahim, H.: Recent survey on crowd density estimation and counting for visual surveillance. Eng. Appl. Art. Intell. 41, 103-114 (2015). https://doi.org/10.1016/ j.engappai.2015.01.007

91. Schaffer, D., Neto, A.B., Antonitsch, A., Musse, S.R.: Towards animating virtual humans in flooded environments. In: ACM SIGGRAPH Conference - Motion in Games, p. 1 (2020)

92. Shi, X., Xue, S., Feliciani, C., Shiwakoti, N., Lin, J., Li, D., Ye, Z.: Verifying the applicability of a pedestrian simulation model to reproduce the effect of exit design on egress flow under normal and emergency conditions. Physica A: Statistical Mechanics and its Applications 562, 125,347 (2021). https://doi.org/10.1016/j. physa.2020.125347

93. Shoulson, A., Marshak, N., Kapadia, M., Badler, N.I.: Adapt: The agent development and prototyping testbed. In: Proceedings of the ACM SIGGRAPH Symposium on Interactive 3D Graphics and Games, I3D '13, p. 9-18. Association for Computing Machinery, New York, NY, USA (2013). https://doi.org/10.1145/2448196. 2448198

94. Stüvel, S.A., Magnenat-Thalmann, N., Thalmann, D., Stappen, A.F., Egges, A.: Torso crowds. IEEE Trans. Visual. Comput. Graph. 23(7), 1823-1837 (2017). https://doi.org/10.1109/TVCG. 2016.2545670 
95. Sun, J., Zhang, J., Li, Q., Yi, X., Liang, Y., Zheng, Y.: Predicting citywide crowd flows in irregular regions using multi-view graph convolutional networks. IEEE Trans. Knowledge Data Eng. (2020). https://doi.org/10.1109/TKDE.2020.3008774

96. Sung, M., Kovar, L., Gleicher, M.: Fast and accurate goal-directed motion synthesis for crowds. In: Proceedings of the 2005 ACM SIGGRAPH/Eurographics Symposium on Computer Animation, SCA '05, p. 291-300. Association for Computing Machinery, New York, NY, USA (2005). https://doi.org/10.1145/1073368. 1073410

97. Testa, E., Barros, R.C., Musse, S.: Crowdest: a method for estimating (and not simulating) crowd evacuation parameters in generic environments. Visual Comput. 35, 1119-1130 (2019)

98. Thalmann, D., Musse, S.R.: Crowd Simulation. Springer-Verlag, Berlin, Heidelberg (2007)

99. Treuille, A., Cooper, S., Popović, Z.: Continuum crowds. In: ACM SIGGRAPH 2006 Papers, SIGGRAPH '06, p. 1160-1168. Association for Computing Machinery, New York, NY, USA (2006). https://doi.org/10.1145/1179352.1142008

100. Tripathi, G., Singh, K., Vishwakarma, D.K.: Crowd emotion analysis using $2 \mathrm{~d}$ convnets. In: 2020 Third International Conference on Smart Systems and Inventive Technology (ICSSIT), pp. 969-974 (2020). https://doi.org/10.1109/ICSSIT48917.2020.9214208

101. Tu, X., Terzopoulos, D.: Artificial fishes: Physics, locomotion, perception, behavior. In: Proc. SIGGRAPH '94, pp. 43-50 (1994)

102. Volonte, M., Hsu, Y., Liu, K., Mazer, J.P., Wong, S., Babu, S.V.: Effects of interacting with a crowd of emotional virtual humans on users' affective and non-verbal behaviors. In: 2020 IEEE Conference on Virtual Reality and 3D User Interfaces (VR), pp. 293-302 (2020). https://doi.org/10.1109/VR46266.2020.00049

103. Wolinski, D., Guy, J.S., Olivier, A.H., Lin, M., Manocha, D., Pettre, J.: Parameter estimation and comparative evaluation of crowd simulations. Comput. Graph. Forum 33(2), 303-312 (2014). https://doi.org/10.1111/cgf.12328

104. Wong, K.Y., Loscos, C.: Hierarchical path planning for virtual crowds. In: Egges, A., Kamphuis, A., Overmars, M. (eds.) Motion in Games, pp. 43-50. Springer, Berlin Heidelberg (2008)

105. Wong, S., Wang, Y.S., Tang, P.K., Tsai, T.: Optimized evacuation route based on crowd simulation. Comput. Visual Media 3, 243261 (2017)

106. Xie, W., Lee, E.W.M., Li, T., Shi, M., Cao, R., Zhang, Y.: A study of group effects in pedestrian crowd evacuation: experiments, modelling and simulation. Safety Sci. (2021). https://doi. org/10.1016/j.ssci.2020.105029

107. Xu, M., Li, C., Lv, P., Chen, W., Deng, Z., Zhou, B., Manocha, D.: Emotion-based crowd simulation model based on physical strength consumption for emergency scenarios. IEEE Trans. Intell. Trans. Syst. (2020). https://doi.org/10.1109/TITS.2020. 3000607

108. Yang, F., Shabo, J., Qureshi, A., Peters, C.: Do you see groups? the impact of crowd density and viewpoint on the perception of groups. In: Proceedings of the 18th International Conference on Intelligent Virtual Agents, IVA '18, p. 313-318. Association for Computing Machinery, New York, NY, USA (2018). https://doi. org/10.1145/3267851.3267877

109. Yersin, B., Maïm, J., Morini, F., Thalmann, D.: Real-time crowd motion planning. Visual Comput. 24(10), 1432-2315 (2008). https://doi.org/10.1007/s00371-008-0286-0

110. Zhan, B., Monekosso, D.N., Remagnino, P., Velastin, S.A., Xu, L.Q.: Crowd analysis: a survey. Mach. Vision Applicat. 19(5), 345-357 (2008). https://doi.org/10.1007/s00138-008-0132-4

111. Zhang, P., Liu, H., Ding, Y.: Crowd simulation based on constrained and controlled group formation. Visual Comput. 31(1), 1432-2315 (2015). https://doi.org/10.1007/s00371-013-0900-7

112. Zhang, Z., Jia, L.: Optimal guidance strategy for crowd evacuation with multiple exits: a hybrid multiscale modeling approach.
Appl. Math. Modell. 90, 488-504 (2021). https://doi.org/10.1016/ j.apm.2020.08.075

113. Zhao, M., Turner, S.J., Cai, W.: A data-driven crowd simulation model based on clustering and classification. In: 2013 IEEE/ACM 17th International Symposium on Distributed Simulation and Real Time Applications, DS-RT '13, p. 125-134. IEEE Computer Society, USA (2013). https://doi.org/10.1109/DS-RT.2013.21

114. Zhao, R., Hu, Q., Liu, Q., Li, C., Dong, D., Ma, Y.: Panic propagation dynamics of high-density crowd based on information entropy and aw-rascle model. IEEE Trans. Intell. Trans. Syst. 21(10), 4425-4434 (2020). https://doi.org/10.1109/TITS.2019. 2953357

115. Zhou, M., Dong, H., Wang, X., Hu, X., Ge, S.: Modeling and simulation of crowd evacuation with signs at subway platform: A case study of beijing subway stations. IEEE Trans. Intell. Transp. Syst. (2020). https://doi.org/10.1109/TITS.2020.3027542

Publisher's Note Springer Nature remains neutral with regard to jurisdictional claims in published maps and institutional affiliations.

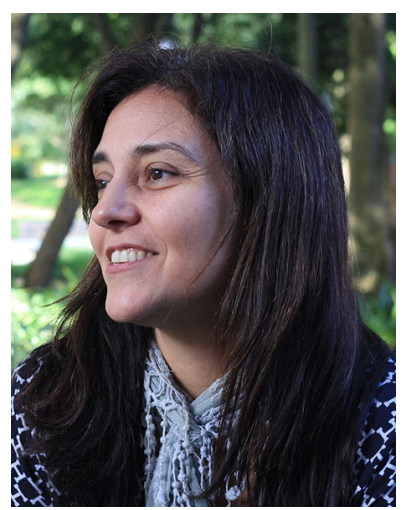

Soraia Raupp Musse is an associate professor at the School of Technology, Pontifical Catholic University of Rio Grande do Sul (Brazil), where she created and still coordinates the Virtual Human Laboratory. Her research interests include Crowd Simulation and Analysis, Facial Animation, and Integration of Computer Graphics, Pattern Recognition and Computer Vision. Dr. Musse has a $\mathrm{Ph} . \mathrm{D}$. in Computer Science from the Ecole Polytechnique Fédérale de Lausanne (Switzerland) where she was supervised by Prof. Daniel Thalmann. Recently, she spent a year at UPENN (USA) working as a visiting scholar with Prof. Norman Badler. She has supervised more than 40 graduate students and postdocs and published more than 160 papers. She also published three books in Crowd Simulation with Springer-Verlag.

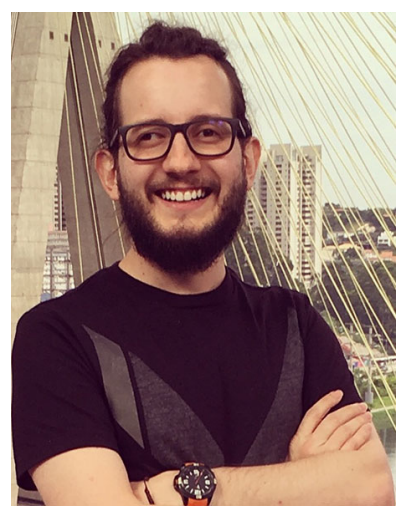

Vinicius Jurinic Cassol received his Ph.D Degree in Computer Science at Pontifícia Universidade Católica do Rio Grande do Sul Brazil, supervised by Prof. Soraia Musse. In addition, during the $\mathrm{PhD}$, he had the opportunity to be a visitor scholar at University of Pensilvania under supervision of Professor Norman Badler. His research focus on crowd simulation and analysis of evacuation process. Vinicius is currently a lecturer for computer graphics and games at UniRitter - Brazil. 


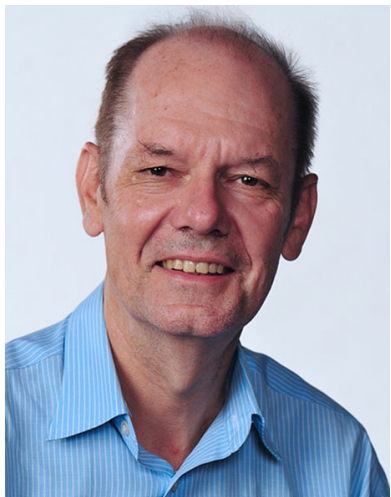

Daniel Thalmann is a Swiss and Canadian Computer Scientist. He is currently Honorary Professor at EPFL and Director of Research development at MIRALab Sarl. Pioneer in research on Virtual Humans, his current research interests include social robots, crowd simulation and Virtual Reality. Daniel Thalmann has been the Founder of The Virtual Reality Lab (VRlab) at EPFL, Switzerland, Professor at The University of Montreal and Visiting Professor/ Researcher at CERN, University of Nebraska, University of Tokyo, and National University of Singapore. From 2009 to 2017, he was Visiting Professor at the Nanyang
Technological University, Singapore. He is coeditor-in-chief of the Journal of Computer Animation and Virtual Worlds, and member of the editorial board of 12 other journals. Daniel Thalmann was member of numerous Program Committees, Program Chair and CoChair of several conferences including IEEE VR, ACM VRST, and ACM VRCAI. Daniel Thalmann has published more than 600 papers in Graphics, Animation, and Virtual Reality. He is coeditor of 30 books, and coauthor of several books including 'Crowd Simulation' (second edition 2012) and 'Stepping Into Virtual Reality' (2007), published by Springer. He received his $\mathrm{PhD}$ in Computer Science in 1977 from the University of Geneva and an Honorary Doctorate (Honoris Causa) from University Paul- Sabatier in Toulouse, France, in 2003. He also received the Eurographics Distinguished Career Award in 2010, the 2012 Canadian Human Computer Communications Society Achievement Award, and the CGI 2015 Career Achievement. Wikipedia: http://en.wikipedia.org/wiki/Daniel_Thalmann 